\title{
Resiliencia, identidad y reconocimiento. Nuevas aproximaciones del concepto a los aportes de George Mead y Axel Honneth
}

\author{
Resilience, identity and recognition \\ New approaches to the concept of the contributions \\ of George Mead and Axel Honneth
}

\author{
Max Telias Delpiano \\ Licenciado en Trabajo Social, (c) Magíster en Trabajo Social. Pontificia Universidad Católica de Chile \\ Correo electrónico: max.telias@gmail.com
}

\begin{abstract}
Resumen
La resiliencia es un constructo que en su desarrollo en las ciencias sociales ha permitido describir situaciones de superación experimentadas por sujetos frente a eventos que, presumiblemente, provocarían un daño en su identidad. En este sentido, múltiples han sido los aportes trabajados sobre esta línea, alcanzando nociones sobre resiliencia que van más allá de las expresiones de la personalidad. Asi, hoy nos enfrentamos a constructos como resiliencia comunitaria, resiliencia familiar y resiliencia empresarial (por nombrar algunos), los cuales pretenden describir fenómenos de superación ante eventos traumáticos vividos por diferentes formas de organización social. Ahora bien, los aportes en esta dirección cargan con ciertas limitaciones metodológicas que, hasta el momento, no han tenido un análisis consistente. En este sentido, el artículo aborda el fenómeno de la resiliencia desde una perspectiva crítica, donde pretende encontrar en los aportes sobre el Reconocimiento desarrollados por Axel Honneth, un espacio de relectura.
\end{abstract}

Palabras clave: Resiliencia, Reconocimiento, Identidad, Honneth

\begin{abstract}
Summary
Resilience is a construct that in social sciences has allowed to describe overcoming situations experienced by subjects facing events that presumably would cause damage to their identity. In this sense, many contributions have been worked on this line, reaching notions of resilience beyond expressions of personality. So, today we can find community resilience constructs, family resilience and business resilience (to name a few), which attempt to describe phenomena of overcoming traumatic events on different forms of social organization. However, the contributions in this direction appear to have methodological limitations, which have no $t$ been consistently analyzed. This article discusses the resilience from a critical perspective, seeking to find in the contributions about the Recognition of Axel Honneth a reinterpretation of this phenomenon.
\end{abstract}

Key words: Resilience, Recognition, Identity, Honneth.

\section{Introducción}

Múltiples sucesos acontecidos en los últimos dos años llaman a reflexionar, pese al desarrollo incuestionable de los modelos descriptivos y acciones preventivas, sobre la imposibilidad de manipular de forma racional situaciones potencialmente nocivas para las personas; nos hacen preguntar qué tan preparados estamos, qué tan predecibles son, qué tan catastróficos pueden ser: en definitiva, qué tan seguros nos sentimos en un contexto histórico en el cual las fuerzas de la naturaleza constantemen- te muestran lo frágiles que somos, haciendo de sí un recordatorio de aquello que Zygmunt Bauman (2005) fundamenta como característica particular del sujeto contemporáneo, el miedo cósmico. Al respecto, difícil es definir un acontecimiento natural (o social) como catástrofe si no es a partir de las consecuencias devastadoras que para sí tiene la consciencia que los aprehende: en otras palabras, aquella sensación de finitud espacio-temporal solo encuentra su condición en el momento en que el desastre se traduce en fenómenos que impactan de 
forma trágica y directa la forma de comprender la vida humana. De esta manera, es posible asumir que son solo las estrategias que fortalecen la experiencia de seguridad aquellas que pueden mitigar, experimentado el suceso, la percepción agobiante de finitud.

De esta manera, surgen formas de abarcar los procesos acontecidos a partir del estudio de aquellas actitudes manifiestas de los sujetos afectados, identificando, comprendiendo y fortaleciendo ciertos posicionamientos valorativos en torno a la apreciación de un fenómeno natural y/o social en particular, con el objeto de enmarcarlos dentro de ciertos contextos que favorecerían o dificultarían la emergencia de dichas actitudes. Tales son los estudios en relación al riesgo, la condición de vulnerabilidad y, el que nos interesa en este artículo, la resiliencia de los sujetos, todos constructos que pretenden organizar, a través del estudio de la experiencia particular de un suceso catastrófico, las acciones desarrolladas por medio de criterios conceptuales que sustentan y organizan un prisma de análisis que -determinado el caso- buscan prevenir condiciones de daño, mitigar situaciones dolorosas, o fortalecer las herramientas con que los sujetos responden a una experiencia de catástrofe.

Pertinente es entonces para el contexto actual intentar analizar aquellos aportes conceptuales que contribuyan, de forma directa o indirecta, a comprender aquellos sucesos que han marcado a Chile en los últimos dos años, dentro de los cuales el terremoto y tsunami acontecidos en febrero de 2010 toman posición como aquellos de los que ha impactado de mayor manera la percepción de sí de los chilenos. Y si bien los motivos por los cuales este suceso en un momento determinado fue un centro de interés mediático para los distintos grupos de interés, y claramente sin cuestionar las consecuencias nefastas que tuvo (y aún tiene) para aquellas personas directamente involucradas, ambos dan pie para poner en discusión un tema que hoy parece central, aún más allá de sus propio aclance: la capacidad que tienen las personas para superar sucesos catastróficos y continuar sin traumas su vida; en otras palabras, la capacidad resiliente que presentan las personas afectadas tanto por el terremoto como por el tsunami.

El artículo comienza realizando una revisión general de las múltiples comprensiones de resiliencia, mostrando cuáles han sido sus principales aportes y dificultades, para luego una realizar una relectura de los presupuestos del constructo a partir de las nociones de la conformación de la personalidad de- sarrollada por George Mead, para culminar en una tercera y última sección con un posible marco de interpretación de la resiliencia desde la perspectiva de Reconocimiento que reinterpreta Axel Honneth a partir de los aportes de Mead y Hegel.

\section{Desarrollo y emergencia de la resiliencia: de sus fundamentos y fronteras}

La resiliencia, en términos simplistas, puede ser comprendida como la capacidad de sobreponerse a un escenario de daño, logrando superar de forma exitosa aquellas situaciones que constituyen un alto riesgo para la integridad personal. Los aportes que este constructo ha realizado a las ciencias sociales son indiscutibles y lo han posicionado dentro de análisis de los más diversos fenómenos. Tal ha sido el avance, que en los últimos años múltiples autores han optado por ampliar las posibilidades analíticas del concepto al utilizarlo para referirse no solo a individuos, sino también a comunidades, familias, y empresas. Esta utilización en sí no conforma un problema, a menos que en su definición se apele a características propias de lo colectivo desde dimensiones que han emergido desde la resiliencia individual, sin hacerse cargo de las implicancias teóricas y prácticas que dicha transposición acarrea. Por otra parte, un número importante de las definiciones de resiliencia refieren a la dimensión contextual y colectiva que influiría en la resiliencia individual, pero sin problematizar las implicancias para la emergencia de la resiliencia más allá de consecuencias directas de estímulos del entorno. Y precisamente, con la pretensión de contribuir a posicionar el constructo de forma más robusta, es que se pretende releer a la resiliencia desde marcos que respondan a conformaciones colectivas de identidad, lo cual, además de otorgar un fundamento diferente, llama a comprender un nuevo origen del concepto.

Considerando lo expuesto, la resiliencia, en términos etimológicos, tiene su origen en el concepto latín de resilio, el cual significa volver atrás, volver de un salto, resaltar (Muñoz \& De Pedro, 2005). Su aplicación dentro de la ciencia moderna se produce inicialmente en las ciencias biológicas, proveniente de la castellanización de la palabra resilience, o resiliency, buscando con ella dar cuenta de la capacidad de un cuerpo para recuperar su tamaño y forma original después de haber sido comprimido, doblado o estirado, o bien la capacidad que presentaría un cuerpo para ajustarse al cambio o a la mala fortuna (Kalawski \& Haz, 2003). Las primeras apariciones del concepto en las ciencias sociales 
surgen dentro de la psicología, las que -asociadas fuertemente a la psiquiatría- emergen en estudios que pretenden expresar que situaciones peligrosas para la vida no afectaban, como era de esperar, a los niños y niñas, mientras que sí lo hacían fenómenos como el desarraigo familiar, optando así por la resiliencia para explicar por qué algunos niños, habiendo sufrido situaciones que implicarían un alto estrés, eran capaces de sobrepasar las adversidades y convertirse en niños y niñas saludables (Menville, 1994). Importante es mencionar que, en un inicio, se buscó vincular el concepto de resiliencia al de invulnerabilidad (Anthony, 1974), queriendo explicar que los niños y niñas, pese a haber estado expuestos a amplios períodos de estrés psicológico, contaban con salud emocional para continuar normalmente su vida. Esta asociación prontamente perdió sustento, puesto que esta implicaría, por una parte, resistencia absoluta al daño y, por otra, la aplicación de la catacterística a todas las circunstancias de riesgo, configurándose necesariamente, en términos genéricos, como un rasgo estable en el tiempo (Kalawski \& Haz, 2003). Esto queda de manifiesto ya en las argumentaciones de Rutter (1985), quien a través de estudios empíricos, fue capaz de argüir que los presupuestos de inexistencia de límites para soportar múltiples formas de sufrimiento, es decir, la inmunidad al daño, estaban completamente alejadas de la realidad, llamando a comprender que la resiliencia está lejos de la ausencia de vulnerabilidad (Waller, 2001). Para dicho autor, la resiliencia podría comprenderse como una relativa resistencia manifestada por algunas personas al enfrentar situaciones consideradas potencialmente riesgosas en términos psicosociales para su funcionamiento o desenvolvimiento, siendo siempre resultante de una interacción de factores genéticos y ambientales, los cuales, a su vez, oscilan en su función, pudiendo actuar como elementos de protección en ciertos momentos y de riesgo en otros (Rutter, 1999). De la forma expuesta, para poder realizar estudios sobre resiliencia, se haría imprescindible conocer las interacciones que establecen aquellas personas que presentan actitudes resilientes con aquellas que no, buscando comprender $-\mathrm{y}$ considerando como central el contexto en que acontece el suceso y el momento histórico vivido por las personas-, cómo es que ambos influirían de diferente forma en relación al enfrentamiento de la adversidad (Santos da Silva, Elsen, \& Lacharité, 2003).

Continuando con esta argumentación, tal y como lo expresan diversos estudios (Barlach, Limongi-França, \& Malvezzi, 2008), el concepto de resiliencia tomó fuerza cuando se le vinculó al de riesgo (Infante, 2005) produciendo un alejamiento de la comprensión del fenómeno en cuanto característica de la personalidad, y entendiéndola en el desenvolvimiento mismo de la confrontación a la adversidad. Se fomenta así la comprensión del constructo de forma sistémica, -dirán algunos-, donde se estructure a la resiliencia en función de la relación individuo-entorno (Waller, 2001; Barlach, Limongi-França, \& Malvezzi, 2008), y no de una capacidad psíquica en particular que presentarían algunos sujetos.

Se aclara entonces que además de un origen biológico y físico, había un origen del concepto desde la psicología, el cual -sin aproximarse a las nociones de invulerabilidad-hacía referencia a la capacidad de las personas de resistir a situaciones adversas sin perder su equilibrio inicial, esto es, la capacidad de acomodarse y reequilibrarse constantemente ( $\mathrm{Ne}$ mer, 2004). Lo anterior tendría fundamento en la capacidad que presentarían los sujetos para movilizar y activar las capacidades de ser, estar, tener, poder y querer, en otras palabras, en la capacidad de la autorregulación y el autoestima (Tavares, 2001). Según lo expuesto, la resiliencia se caracterizaría desde sus inicios por un conjunto de procesos sociales e intrapsíquicos que posibilitarían tener una vida sana viviendo en un contexto insano (Muñoz $\&$ De Pedro, 2005), donde son los procesos relacionales que se dan entre el individuo y su entorno los que posibilitarían el desarrollo de actitudes resilientes (Chaskin, 2008). En este sentido, la resiliencia sería una característica esencialmente dinámica, la cual, a su vez, variaría en el tiempo ante diferentes circunstancias, siendo el resultado de un equilibrio entre factores protectores, factores de riesgo, y personalidad del individuo (Muñoz $\&$ De Pedro, 2005). Lo anterior se contrapondría a una perspectiva que propone a la resiliencia como característica refleja únicamente de elementos individuales, como lo es el considerar, en cuanto dimensión excluyente del concepto, la capacidad de las personas de resistir a situaciones adversas sin perder su equilibrio inicial, en otras palabras, la capaciad de acomodarse y re-equilibrarse constantemente ante cualquier potencial contexto dañino (Nemer, 2004). Con esta concepcion se perdería el foco puesto en la relación que presentaría la resiliencia, más que una característica estable del individuo, esta emergería ante la flexibilidad interna que permitiría interactuar con éxito confrontaciones del mundo exterior consideradas nocivas para el individuo (Nemer, 2004; Schiera, 2005) 
Expuesto de esta forma, cabe mencionar que en la medida en que el constructo fue robusteciendo los análisis en esta línea, y como es de esperar considerando los pasos alcanzados, se vio en la necesidad de vincular de forma más clara aquellas capacidades que los niños y niñas (ahora sujetos) presentaban en relación a los contextos en que se desarrollaban, haciendo de manifiesto que la resiliencia emergería únicamente en un marco de dinamismo (Nemer, 2004), el cual requeriría considerar como central la relación del individuo con su entorno. En este proceso, el lenguaje con el cual el sujeto aprehendía las condiciones de su contexto adquiriría entonces un rol fundamental, puesto que a través de él, el sujeto sería capaz de interpretar y aprehender su entorno social y cultural, codificando signos que le son reconocibles para generar respuestas ante situaciones extremas. La resiliencia entonces podría ser comprendida de forma inicial como un concepto que apela a una serie de contestaciones lingüísticas, internas del individuo, que surgirían a partir de una reflexión que facilitaría la emergencia de actitudes que lo fortalezcan luego de un estrés traumático (Cyrulnik, 2002), donde las conexiones tanto con otros como con el entorno se configurarían como elementos centrales del propio bienestar de sí (Maybery, Pope, Hodgins, Hitchenor, \& Shepherd, 2009).

En el sentido expuesto, y precisamente en la forma de comprender esta vinculación, el concepto de resiliencia ha sido problematizado a partir de su emergencia en cuanto respuesta propia de la individualidad del sujeto, la cual se generaría a partir de una relación de negatividad con un entorno considerado difícil, es decir, se crearía en cuanto respuesta contradictoria a una situación externa al individuo que genera estrés sobre él (Schiera, 2005). Así, y si bien la mediación paradójica del constructo permitiría dar respuestas antagónicas a las esperadas producto del trauma, dichas respuestas son consideradas desde la consciencia del sujeto, desde respuestas que se crean a partir de la forma en que reacciona su personalidad ante un contexto dañino para su autocomprensión de seguridad. Esta situación ha sido trabajada por muchísimos estudios, los cuales identifican una multiplicidad incalculable de factores protectores y de riesgo que influirían, para bien o para mal, en la conformación de actitudes resilientes (Norris \& Stevens, 2008; Suarez-Ojeda \& Melillo; Cyrulnik, 2002; entre otros).

Ahora bien, cabe destacar que su expansión hacia las ciencias sociales ha culminado con avanzar hacia distintos niveles de uso, pudiendo observar hoy cómo se aplica el concepto de resiliencia tanto al individuo como a grupos, tratando de forma indiferenciada los usos del constructo a nivel de grupos, familias y comunidades resilientes (Atheortúa, 2002; Benghozi, 2005; Suarez-Ojeda \& Melillo, 2001; por solo nombrar algunos). Esta transposición si bien no constituye en sí un problema, comprende ciertos supuestos que figuran a partir de comprensiones ontológicas de la personalidad que no siempre responden a las lógicas en las cuales se exponen -como se ha manifestado- las nociones de resiliencia, las que, poniendo el foco en lo fundamental que constituye la categoría de lo colectivo en las potenciales asociaciones en pos de la superación de un desastre (Tse \& Liew, 2004; Suarez-Ojeda \& Melillo, 2001), culminan por romper dicho énfasis al tratar indistintamente los componentes de la resiliencia de los individuos y de lo colectivo de la comunidad, familia u otra forma de asociación. Lo anterior se sustenta en la medida en que se pretende construir la noción de resiliencia a partir del rol central que presentaría la relación del individuo con su entorno, sin desarrollar aquellas nociones de la configuración de la personalidad a partir de su vínculo con los demás: en otras palabras, cuando se pretende justificar a la resiliencia como característica dinámica tomando como noción central de la constitución de la persona aquellas perspectivas que posicionan al individuo como ente nuclear de la sociedad. Este tipo de argumentaciones tienen de base la primacía del individuo, sustentando las nociones de sociedad en las bases de la teoría social del contrato, defendiendo que "los individuos existen previamente como individuos inteligentes, como personas, y que dichos individuos se reúnen y forman la sociedad" (Mead, 1953, p. 254), y que serían estas personas que de forma individual podrían desarrollar, en la medida en que se encuentren en un entorno que las estimule, actitudes resilientes. Una forma de ceguera sería no considerar que estas perspectivas ponen una real importancia en aquellos mecanismos que del medio surgen para la modificación de la personalidad, pero ingenuo sería no considerar que en el proceso de la formación de la personalidad la comunicación presenta un rol fundante, en cuanto el individuo adquiere su persona únicamente a través de la interacción con otros, solo gracias a la formación de los procesos sociales mediante la comunicación significante, dejando de manifiesto, si se es consistente con la argumentación, que la persona no podría encontrarse antes de la sociedad, sino que esta debe existir de forma que la defina (Mead, 1953). 


\section{George Mead y Axel Honneth: nuevos horizontes desde viejos orígenes}

Asumiendo lo expuesto en la sección anterior, la referencia a la resiliencia como una característica (dinámica) de la personalidad en torno a las reacciones que se desenvuelven en un contexto dañino, conlleva la alusión a las autocomprensiones de la personalidad, las que surgen únicamente en torno al proceso de comunicación desarrollado en la conformación de la misma, es decir, indisociables del proceso de socialización de los sujetos en cuestión, donde las posibilidades de acción que emergen a partir de la generalización de las expectativas normativas de comportamiento identificadas en los distintos compañeros de interacción, modifican las posibles representaciones sociales en las que el sujeto despliega sus potenciales acciones (Honneth, 1997). Y es justamente con la pretensión de contribuir a una problematización de la resiliencia a niveles de análisis que ya no respondan a una carga fundamentalmente individual, en cuanto a asumir a la sociedad como una asociación entre diferentes individuos, que se busca enfocar los aportes del constructo desde nociones que tomen distancia de una filosofía de la conciencia, realizando hincapié en una conformación del espacio colectivo desde nociones referentes a la construcción del espacio de interacción de forma intersubjetiva. En otras palabras, se busca poner como posición ontológicosocial aquella comprensión del espacio que dice de su construcción a partir de la configuración de la identidad de los sujetos en torno a la experiencia (mediada comunicativamente) del enfrentamiento a un otro, donde proyectamos de nosotros aquellas aprehensiones colectivas que hacen de complemento de mi individualidad y que, producto del proceso de socialización, se van cedimentando en la configuración personal (Mead, 1953). Es posible decir, además, que dicho proceso toma forma a través de los discursos lingüísticos que ejercen los sujetos que, para el caso de la resiliencia, comunicarían sus expectativas y proyecciones a partir de una situación de catástrofe, la cual sería única y exclusivamente explicable por las codificaciones lingüísticas que, a su vez, serían solo comprensibles a partir del propio lenguaje construido en el proceso de configuración de identidad colectiva. Esto provoca una escisión en la figura epistemológica de entender al sujeto dotado de una consciencia que comprende (la idea de consciencia individual, responsable de actitudes resilientes), es decir, la comprensión del mundo ya no se lograría a través de la acción de la consciencia, sino del comprender que los significados se captan del propio lenguaje, "pues lo que la proposición significa es dicho de nuevo por otra proposición" (Wittgenstein, 1969, p. 75), perdiendo sentido una posición explicativa de lo social que tome de base la asociación de individuos.

Pero la forma en que los sujetos desarrollan su personalidad no solo responde a expresiones del lenguaje propiamente tal, sino al proceso de comunicación completo en cuanto expresión significante, en otras palabras, al complejo entramado de relaciones sociales que moldean, por verbalizar someramente, al individuo y a la comunidad. Ahora bien, conviene aclarar que la comunicación como proceso no puede comprenderse como algo existente por sí mismo, o como presupuesto del proceso social, sino que la relación social es presupuesta a fin de hacer posible la comunicación y el pensamiento, el cual no es otra cosa que "la reacción del individuo a la actitud del otro, en el amplio proceso social en el que se encuentran ambos involucrados, y la dirección de la acción anticipatoria de uno mismo por medio de las actitudes de los otros que uno adopta" (Mead, 1953, p. 277), haciendo del reconocimiento un constructo central en la conformación de la personalidad. Con esto, el distanciamiento de aquellas nociones que comprenden el origen de la resiliencia (individual, comunitaria, etc.) como aquel proceso dinámico por el cual un individuo es capaz de sobrepasar las adversidades de un entorno que lo daña y a la vez lo estimula, queda expuesta en el sentido en que ya no se trataría de comprender aquellos elementos de la personalidad y del entorno que facilitan u obstaculizan las actitudes resilientes, sino antes bien de conocer cuáles han sido los procesos sociales, en cuanto pensamiento y comunicación, que dinamizan la expectativa internalizada del otro generalizado (utilizando la nomenclatura de Mead) que provoca en el individuo la adopción de ese papel, es decir, que hace volver sobre sí y dirigir la comunicación en torno al restablecimiento y/o superación de situaciones que son consideradas nocivas para la comunidad. Y si bien los aportes expuestos hasta acá fundamentan cómo comprender a la resiliencia desde nuevos cimientos, aún no queda claro el anclaje de la resiliencia trabajada hasta hoy y las formas de interpretación explicitadas.

¿Cómo comenzar, entonces, un posicionamiento del constructo de la resiliencia que, haciéndose cargo de las presuposiciones expuestas, abra paso a nuevas constelaciones? Posiblemente un punto de partida sea el considerar el origen práctico del desplante de la actitud resiliente: la experiencia de sufrimiento. Gran parte de los múltiples aportes so- 
bre resiliencia manifestados en el apartado inicial -que por cierto son indudables- sobre el posicionamiento del constructo, toman sustento en que es a partir de una experiencia de sufrimiento a la que los sujetos han sido capaces de sobreponerse, el espacio donde la resiliencia tendría lugar; se busca identificar aquellas condiciones, tanto externas como internas al sujeto, que facilitarían la apropiación de expectativas y proyecciones de superación delimitadas a una experiencia potencialmente dañina de una situación en particular, aprehendiendo las mejores posibilidades que en un determinado momento histórico se aprecian a partir de una vivencia trágica de sufrimiento. Ahora bien, esta experiencia tiene su origen en la imposibilidad de desarrollar un proceso de autorrealización en condiciones sociales complejas, que muchas veces son agravadas por acontecimientos catastróficos.

Siendo así, el sufrimiento respondería a una experiencia de restricción de las facultades, racionalmente constituidas, de autorrealización (Honneth, 2009), haciendo de la resiliencia un constructo indisociable de aquellas patologías que presentaría la sociedad contemporánea que inhibirían el potencial de desarrollo de los sujetos. Aquella perspectiva teórica que se hace cargo de las críticas expuestas en torno a la configuración de la personalidad de forma colectiva en cuanto asume la idea de que "una autorrealización cooperativa de los sujetos no podrá ser alcanzada sin un núcleo de convicciones de valor compartidas" (Honneth, 2009a, p. 36), unifica la noción de sufrimiento con las condiciones sociales que imposibilitan el desarrollo de los sujetos, es la Teoría Crítica, la cual, desde la perspectiva de Honneth, vincula los constructos de identidad, reconocimiento y agravio moral a partir de un legado intelectual que pretende comprender aquella expresión del sufrimiento subjetivo en torno a las experiencias que hacen persistir en los sujetos un interés por lograr una autorrealización, pese a todas las deformaciones de la racionalidad social (Honneth, 2009b). ¿Cómo comprender, entonces, una aproximación de las nociones de resiliencia, que parecieran estar ancladas a figuras psicológicas individualistas, a una perspectiva de la Teoría Crítica fundamentada en el posicionamiento de Axel Honneth? ¿Qué criterios, lineamientos y conceptos se deberían, a modo inicial, comprender para una resiliencia no-individual que se haga cargo de éstos aportes?

\section{Resiliencia y reconocimiento: aproximaciones teóricas}

Precisamente en función de realizar dichas aproximaciones entre las experiencias traumáticas de aquellos sujetos que dinamizan discursos dentro de un contexto particular, y aquellas expresiones intersubjetivas que permiten sobrellevar dichas situaciones, es que emergen diferentes posibilidades de aproximación teórica. Y es aquí donde toma lugar la propuesta explicitada en torno al Agravio Moral desarrollada por Axel Honneth (2009), la cual se posiciona como espectro conceptual que reconfigura las proposiciones realizadas por Hegel y Mead en torno al concepto de reconocimiento. Y producto de la adopción y reinterpretación de dichos aportes, es que esta perspectiva no se constriñe a los límites de una conciencia individualizada como fundamento último del actuar social, tal y como se exponen los aportes tradicionales sobre la resiliencia, sino que asume que para todo análisis filosófico y social no se puede partir de las acciones realizadas por sujetos aislados entre sí, sino por los vínculos morales en los que siempre se han movido en común: "se pretende suponer como especie de base natural de la socialización humana un estado que siempre ha estado caracterizado por la existencia de formas elementales de convivencia intersubjeiva" (Honneth, 2009c, p. 209). Puesto de esta manera, no son los intereses particulares los que corresponden al fundamento último de la sociedad, antes bien, son los vínculos morales que establecen dichos sujetos los que dan cabida al movimiento incesante de lo múltiples fenómenos sociales, incluidas en ellos las manifestaciones resilientes.

Con estos elementos, ¿cómo se plasman los sujetos ante los diferentes escenarios que toman vida en los contextos sociales? El posicionamiento de los sujetos en dichos movimientos se da en un espacio dialógico, en parte abierto y en parte interno, con los demás, y de aquí que en el proceso de configuración de identidad el reconocimiento adquiera una gran importancia, dado que la "propia identidad depende, en definitiva, de mis relaciones dialógicas con los demás" (Taylor, 2000, p. 65). Al respecto, la configuración de la identidad de los sujetos no se encuentra, en términos generales, vinculada de forma positiva al reconocimiento, antes bien se asociaría de forma negativa a tres estratos de la relación práctica que establecen dichos sujetos consigo mismos, es decir, a tres momentos (en ningún caso temporales) asociados al sentimiento que una persona posee de sí misma con respecto a las capacidades y derechos que le corresponden dentro de un tiempo 
y espacio socialmente establecido por ella: la confianza en sí mismo, vinculada a las necesidades y deseos físicos primarios; la estimación de sí mismo, vinculada a la conciencia de ser un sujetos moralmente responsable; y el sentimiento de valer por sí mismo, asociado a la conciencia de poseer buenas y valiosas capacidades (Honneth, 2009c). Aquella formalización del establecimiento de la identidad se posiciona como prefiguración normativa de condiciones necesarias en las relaciones sociales, de aquí la exigencia moral de reconocimiento en torno a las múltiples posibilidades de relación social que puedan presentar los sujetos.

De esta forma, el considerar de manera positiva y a priori aquellos mecanismos por los cuales las relaciones sociales se hacen cargo de las prestaciones que provocan las dinámicas de reconocimiento, constituiría un mecanismo formal que no haría otra cosa que alejarse de la dinámica constitutiva de identididad, por lo cual únicamente es posible hacerlo a través de aproximaciones negativas al problema del reconocimiento. Así pues, es posible aseverar que "a cada estrato de la relación práctica consigo mismo corresponde entonces un tipo particular de injusticia, que a su vez se corresponde con un grado específico de agravio psíquico" (Honneth, 2009d, p. 320): en otras palabras, a la configuración fáctica de la identidad colectiva se encontrarían fuertemente vinculadas expresiones que tomen posición como experiencia de sufrimiento. De la forma expuesta, es que puede entrar en juego la resiliencia vista ya no solo como una expresión del individuo y su contexto, sino que como una respuesta positiva ante configuraciones agraviantes que se asocien a alguno de los diferentes estratos de la relación práctica consigo mismos que, a partir de un mecanismo de auto-observación racional de las propias facultades de realización personal, se encuentren en el desenvolvimiento mismo de la configuración de la identidad.

Sin entrar profundamente a desarrollar aquí dichas aproximaciones, cabe retomar que cada una de las clases de injusticia así obtenidas debe su caracterísica al valor, con respecto al cual un sujeto es despreciado o no reconocido en tres ámbitos de su personalidad: vulneraciones morales que le roban a una persona la seguridad de poder disponer de su bienestar físico y psíquico; vulneraciones morales cuya caracterísica es el desprecio de la responsabilidad moral que las personas tienen por sus acciones; y vulneraciones morales que pretenden, por medio de la humillación y la falta de respeto, que las capacidades de una o varias personas no gocen de ningún reconocimiento (Honneth, 2009d). De esta forma, es posible considerar que lo que subyace en términos de motivación a la actitud de protesta social de aquellos sectores sociales desventajados es la experiencia de la violación de ideas de justicia intuitivamente dadas, "y el núcleo normativo de semejantes ideas de justicia lo constituyen una y otra vez las expectativas asociadas al respeto, a la dignidad, al honor o a la integridad propios." (Honneth, 2009e, p. 261). Al respecto, es posible pensar que los problemas en las relaciones de reconocimiento se vincularían primordialmente a un impedimento en la autorrealización personal, al distorsionar la relación práctica del yo del sujeto en relación a su comunidad, dejando los problemas relativos a lo justo en segundo plano. Siendo así, habría que replantear el reconocimiento como un problema de justicia, argumentando que "es injusto que a algunos individuos y grupos se les niegue el estatus de interlocutores plenos en la interacción social como consecuencia solo de unos patrones institucionalizados de valor cultural en cuya elaboración no han participado en pie de igualdad" (Fraser, 2003, p. 36). En este sentido, se pondría de manifiesto el carácter bidimensional que presentarían todas las relaciones de subordinación, puesto que por una parte se asociarían a patrones institucionalizados de valor cultural que impiden la paridad en la participación, problemas relativos al reconocimiento, mientras que por otra se vincularían a patrones de desigualdad en las relaciones económicas, apelando a problemas de redistribución, rompiendo así con la falsa dicotomía expuesta entre ambos constructos (Fraser, 2003).

Considerando entonces las distinciones entre reconocimiento y redistribución expuestas en el párrafo anterior, conviene manifestar que el sostener dicha paridad conceptual es expresión de una importante tendencia a reducir la lucha por el reconocimiento a la identidad cultural, cuestión que correspondería, por una parte, a una simplificación histórica en cuanto pretende entender la totalidad de las luchas por el reconocimiento desarrolladas en la modernidad a un único aspecto exclusivo y, por otra, a una reducción conceptual, puesto que el reconocimiento no es solo una cuestión cultural, sino que se encuentra vinculada a derechos y dinámicas familiares, entre otras dimensiones. En otras palabras, el reconocimiento debe ser abarcado como un fenómeno diferenciado de naturaleza moral, así como una acción social, donde "la gramática moral viene a decir que los puntos de referencia morales del reconocimiento pueden ser muy distintos y solo 
en una pequeña medida pueden dar cabida a algo así como la peculiaridad moral de un colectivo" (Honneth, 2009f, pp. 50-51). Así, y en relación a la redistribución, las dinámicas de distribución no se encuentran vinculadas únicamente a relaciones de producción, sino que deben ser comprendidas como expresión institucional del dispositivo sociocultural que determina en un momento histórico particular qué grado de apreciación presentan ciertas actividades, dejando de manifiesto que la propia lucha por la distribución se halla enraizada en una lucha por el reconocimiento (Honneth, 2010).

Son las luchas por el reconocimiento entonces aquellas figuras capaces de vincular las experiencias de agravio moral con aquellas expresiones, tanto individuales como colectivas, que buscan la reivindicación de las experiencias subjetivas dañadas por patrones institucionalizados de relación social. De aquí la pertinencia de vincular las concepciones correspondientes a la perspectiva de reconocimiento otorgada por Axel Honneth y las nociones de resiliencia reelaboradas desde la apropiación de las críticas manifiestas, puesto que encuentran su espacio en aquellas experiencias de vulneración asociadas a la configuración de identidad intersubjetivamente construida, de las cuales emergen múltiples respuestas que permiten a los sujetos configurar luchas, gramáticamente determinadas, en torno a la restitución y reconocimiento de su identidad.

En este sentido, la resiliencia como manifestación social podría ser comprendida bajo el marco de las luchas por el reconocimiento, en cuanto permite posicionarla como un mecanismo de respuesta ante experiencias de vulneración en la autocomprensión de sí mismo (representada por los tres niveles de la relación práctica), que, teniendo como origen aquella vivencia de sufrimiento, muestran de qué manera las múltiples internalizaciones de los comportamientos colectivos tienen repercusiones en las posibles reacciones del sujeto ante una situación social dinamizada por una catástrofe, las que, sustentadas en apreciaciones normativas histórica y localmente determinadas, otorgan valor a ciertas formas de resistencia. Siendo así, la resiliencia pasaría a tomar posición como un constructo que permitiría describir, acorde a las lógicas institucionalizadas de valor, un momento de la experiencia que dice de la proximidad de las nociones de autorrealización determinadas por el ideal normativo obstruido por situaciones catastróficas que impactaron sobre la percepción de sí de los sujetos.

\section{Consideraciones para la relectura del constructo}

Como se ha manifestado a lo largo de este artículo, y sin querer abordar el problema más allá de una posible relectura inicial, la resiliencia como constructo ha marcado una línea de avances interpretativos importante dentro de las disciplinas de las ciencias sociales, sobre todo en el área de la psicología. Asumiendo la ventaja de sus aportes, el concepto se ha deslizado hacia una utilización que escapa a las pretenciones psicológicas iniciales, haciendo imprescindible una problematización en su uso. En este sentido, las consecuencias que ha traído consigo la incorporación del comprender el desarrollo de la personalidad de forma colectiva a las diferentes áreas de estudio, pueden mover los cimientos de las formas tradicionales de comprender a la resiliencia, en cuanto se asumía como característica que el sujeto individualmente consciente generaba a partir de una experiencia traumática. El hecho de romper con esta idea, traspasando el desarrollo de actitudes resilientes a las dinámicas discursivas que se generan a partir de la comunicación y de las experiencias que lo anteceden, hace posible la entrada de diversas líneas de interpretación al fenómeno de la resiliencia. Aquí es donde entra en juego el replanteamiento que realiza Honneth del concepto de reconocimiento aportado por Hegel, el cual permite ampliar la concepción psicológico-individal del constructo hacia una configuración colectiva del vínculo moral establecido por los sujetos, el cual, a su vez, estaría permeado por relaciones de agravio moral que provocarían sentimientos de injusticia que, por su parte, impulsarían manifestaciones de reivindicación identitaria, de las cuales la resiliencia podría tomar parte importante.

De esta forma entonces, es posible rescatar que es a partir de aquella experiencia de sufrimiento de la cual los sujetos toman posición en torno a las delimitaciones impuestas a su autorrealización, donde la resiliencia tiene su origen, pues si no se presenta una situación que pueda plasmarse en un potencial daño (físico o simbólico) al desarrollo pleno de su identidad, no habría mecanismo al cual el sujeto se pueda rebelar. Pero esta relación va más allá, pues la resiliencia puede ser leída como un resultado positivo (no necesariamente pasivo, ni necesariamente explícito) de aquellas expresiones sociales de la relación práctica consigomismo que desarrollan dichos sujetos, las cuales están en directa relación con las vulneraciones plasmadas en la conformación de la identidad colectiva y de la apreciación de valor en un determinado marco histórico. En otras palabras, 
la resiliencia encontraría, desde esta perspectiva, su fundamento en aquellas experiencias de sufrimiento que tienen como consecuencia una experiencia de agravio en torno al bienestar físico y psíquico, a la responsabilidad moral de sus acciones y, por último, al reconocimiento de sus capacidades, todos enmarcados dentro de determinadas apreciaciones sociales de valor.

Lo anterior permitiría, a modo de conclusión, observar dos consecuencias para el constructo de la resiliencia: una primera, que diría relación con el comprender a la resiliencia como un mecanismo antiagraviante dentro de la configuración de la identidad y las experiencias de sufrimiento; y una segunda, más importante aún, que obligaría, si lo que se quiere es comprender cómo se expresaría en términos fácticos, a estudiar las determinadas configuraciones locales en torno a la apreciación del valor que tendrían determinadas formas de vulneración. De esta manera, si se quiere conocer cómo se comprende y manifiesta la resiliencia, se haría imprescindible conocer los patrones institucionalizados de reconocimiento (Honneth, 2009e), puesto que toda aproximación externa al problema de la configuración de la identidad colectiva será ciega a aquellas formas de vulneración que los propios sujetos afectados experimentan como sufrimiento. Y si una hay metodología que permite realizar este ejercicio, que queda por razones obvias fuera de las problematizaciones expuestas en este ensayo, es la reconstructiva hermenéutica, la cual permitiría, por una parte, aprehender las normas sociales que expresan al mismo tiempo las forma de racionalización social, mientras que, por otra, controla que en la praxis se siga conservando su significado original (Honneth, 2009g).

Quedan expuestas, llamando a nuevos avances, las siguientes preguntas: ¿cómo podría posicionarse en este marco una perspectiva del reconocimiento que comprenda la amplitud de la conformación de identidad de los sujetos en la actual sociedad chilena? ¿Cuáles podrían ser, a modo de estudio, las dinámicas de agravio que se generan en torno al contexto del terremoto acontecido en Chile el año 2010? ¿Cómo se posicionaría una perspectiva de la resiliencia que, haciéndose cargo de las dinámicas de agravio moral, responda al contexto del terremoto en Chile?

\section{Referencias bibliográficas}

BARLACH, L., LIMONGI-FRANÇA, A. C., \& MALVEZZI, S. (2008). O conceito de Resiliência Aplicado ao Trabalho nas Organizações. Revista Interamericana de Psicologia/Interamerican Journal of Psychology, 42 (1), 101-112.

CYRULNIK, B. (2002). La resiliencia en el curso de la vida: el encuentro con los recursos externos. En B. Cyrulnik, La Resiliencia. Desvictimizar a la víctima (págs. 183-208). Cali, Colombia: Rafue.

FRASER, N. (2003). La justicia social en la era de la política de la identidad: Redistribución, reconocimiento y participación. En N. Fraser, \& A. Honneth, ¿Redistribución o Reconocimiento? (pp. 17-88). Madrid: Morata.

HONNETH, A. (1997). Reconocimiento y Socialización: Mead y la transformación naturalista de la idea de Hegel. En A. Honneth, La Lucha por el Reconocimiento: Por una Gramática Moral de los Conflictos Sociales (págs. 90113). Barcelona, España: Crítica.

HONNETH, A. (2009). Patologías de la Razón. Historia y Actualidad de la Teoría Crítica. Buenos Aires, Argentina: Katz Editores.

HONNETH, A. (2009a). Una patología social de la razón: sobre el legado intelectual de la Teoría Crítica. En A. Honneth, Patologías de la Razón. Historia y actualidad de la Teoría Crítica (págs. 27-51). Buenos Aires, Argentina: Katz editores.

HONNETH, A. (2009b). Crítica del Agravio Moral. Patologías de la Sociedad Contemporánea. Buenos Aires, Argentina: Fondo de Cultura Económica.

HONNETH, A. (2009c). Desarrollo moral y lucha social. Enseñanzas de filosofía social de la obra temprana de Hegel. En A. Honneth, Crítica del Agravio Moral. Patologías de la Sociedad Contemporánea (pp. 197-224). Buenos Aires: Fondo de Cultura Económica.

HONNETH, A. (2009d). Entre Aristóteles y Kant. Esbozo de una Moral del Reconocimiento. En A. Honneth, Crítica del Agravio Moral. Patologías de la Sociedad Contemporánea (pp. 307-332). Buenos Aires: Fondo de Cultura Económica.

HONNETH, A. (2009e). La Dinámica Social del Desprecio. Para determinar la posición de una Teoría Crítica de la Sociedad. En A. Honneth, Crítica del Agravio Moral. Patologías de la Sociedad Contemporánea (pp. 249-274). Buenos Aires: Fondo de Cultura Económica.

HONNETH, A. (2009f). Patologías de lo Social. Tradición y Actualidad de la Filosofía Social. En A. Honneth, Crítica del Agravio Moral. Patologías de la Sociedad Contemporánea (págs. 51-124). Buenos Aires, Argentina: Fondo de Cultura Económica.

HONNETH, A. (2009g). Crítica Reconstructiva de la Sociedad con salvedad Genealógica. Sobre la idea de la "Crítica" en la Escuela de Frankfurt. En A. Honneth, Patologías de la Razón. Historia y actualidad de la Teoría Crítica (págs. 53-63). Barcelona, España: Katz editores. 
HONNETH, A. (2010). Reconocimiento y menosprecio. Sobre la fundamentación normativa de una teoría social. Barcelona: Centro de Cultura Contemporánea de Barcelona.

HONNETH, A. (2009 3-febrero). La lucha por el reconocimiento debe ser considerada un fenómeno claramente diferenciado de naturaleza moral, así como una acción social. (D. Gamper Sachse, Interviewer). Corresponde a una entrevista que está contenida en Honneth, A (2010).

INFANTE, F. (2005). La resiliencia como proceso: una revisión de la literatura reciente. En A. Melillo, \& E. Suárez-Ojeda, Resiliencia: descubriendo las propias fortalezas (págs. 31-53). Buenos Aires, Argentina: Paidós.

KALAWSKI, J., \& HAZ, A. (2003). Y...iDónde está la resiliencia? Una refleción conceptual. Revista interamericana de Psicología, 37 (2), 365-372.

MAYBERY, D., POPE, R., HODGINS, G., HITCHENOR, Y., \& SHEPHERD, A. (2009). Resilience and wellbeing of small inlad communities: community assets as key determinants. Rural Society, 326-339.

MEAD, G. (1953). Espíritu, Persona y Sociedad. Desde el punto de vista del Conductismo Social. Buenos Aires, Argentina: Paidós.

MUÑOZ, V., \& DE PEDRO, F. (2005). Educar para la resiliencia. Un cambio de mirada en la prevención de situaciones de riesgo social. Revista Complutense de Educación, 107-124.

NEMER, D. (2004). A resilência em discussao. Psicologia em Estudio, 67-75.

RUTTER, M. (1999). Resilience concepts and findings: implications for family therapy. Journal of Family Therapy, $21,119-114$.

SANTOS DA SILVA, M. R., ELSEN, I., \& LACHARITÉ, C. (2003). Resiliência: concepções, fatores associados e problemas relativos à construçao do conhecimento na área. Paidéia, 13 (26), 147-156.

SCHIERA, A. (2005). Uso y abuso del concepto de Resiliencia. Revista de Investigación en Psicología, 129-135.

SUÁREZ-OJEDA, E., \& MELILLO, A. (2001). Resiliencia: descubriendo las propias fortalezas. Buenos Aires, Argentina: Paidós.

TAVARES, J. (2001). A resiliência na sociedade emergente. En J. Tavares, Resiliência e educação (págs. 43-76). São Paulo, Brasil.

TAYLOR, C. (2000). La política del reconocimiento. En C. Taylor, El multiculturalismo y la política del reconocimiento (pp. 53-116). México D.F.: Fondo de Cultura Económica.
TSE, S., \& LIEW, T. (2004). New Zeland experiences: how is a community resilience manifested in Asian Communities? International Journal of Mental Health $\mathcal{E}$ Addiction, 1-8.

WALLER, M. A. (2001). Resilience in ecosystemic context: Evolution of the concept. American Journal of Orthopsychiatry, 290-297.

WiTtGenstein, L. (1969). Gramática Filosófica. Oxford: Basil Blackwell. 